\title{
Interaction of yoghurt and orange juice flavanones in an in vitro model of the upper GI tract does not explain reduced urinary excretion of phenolic acids in humans
}

\author{
M. Hou, E. Combet and C.A. Edwards \\ Human Nutrition, School of Medicine, College of Medical, Veterinary \& Life Sciences University of Glasgow, \\ New Lister Building, Glasgow Royal Infirmary, 10-16 Alexandra Parade, Glasgow, G31 2ER, Scotland, UK
}

Orange juice (OJ) flavonoids, mostly flavanones, are poorly bioavailable and extensively metabolised by colonic bacteria to phenolic acids. Roowi et al. reported a six fold reduction of urinary phenolic acids in healthy volunteers, after intake of full fat yogurt (Y) ${ }^{(1)}$, with little insight into the mechanism. Y is mainly digested and absorbed in the small intestine, therefore its impact as part of the food matrix would most likely occur in the upper gastrointestinal tract, reducing the availability of flavanones for colonic bacterial degradation to phenolic acids. Polyphenols have hydrophilic and hydrophobic qualities that may interact with other food ingredients. Interaction of flavanones with lipids and proteins in yoghurt may alter the availability of hesperidin and narirutin in OJ and the rate/speed of their delivery to the colon. Flavanone interactions with Y were examined in an upper GIT model by following the amount of extractable hesperidin and narirutin in digestion fluid with smooth OJ (not from concentrate Tropicana) and OJ with pulp (OJbits; Tropicana with bits). OJ or OJbits $(40 \mathrm{ml})$ and Y $(25 \mathrm{ml}$; home-made with starter culture Streptococcus thermophiles and Lactococcus bulgaricus) were subjected to the two-step of digestion in vitro (gastric phase: pepsin/ $\mathrm{HCl}, \mathrm{pH} 2 \mathrm{for} 1 \mathrm{~h}$; small intestinal phase: pancreatin enzymes/bile salts, $\mathrm{pH} 7$ for $6 \mathrm{~h}$ ). A similar set of incubations were made with a probiotic (Bifidobacterium, Lactobacillus acidophilus, Streptococcus thermophilus) yoghurt. Hesperidin and narirutin in digestion fluid were measured with HPLC-PDA, and total phenol by Folin-Ciocalteau method. No starter culture bacteria survived the acid digestion step (no growth on MRS agar plates).

In the presence of $Y$, total amount of hesperidin and narirutin were 1.5 and 2.1 fold higher in OJ $(p<0.001)$ and OJbits $(p<0.03)$ respectively, after upper GI digestion (control: no Y; Fig 1). Total phenol also was higher in digestion fluid with Y ( $p<0 \cdot 05$; Fig 2). In addition, there was no aglycone detected in the digestive fluids. Similar results of higher hesperidin and narirutin were obtained with probiotic yoghurt (data not shown).

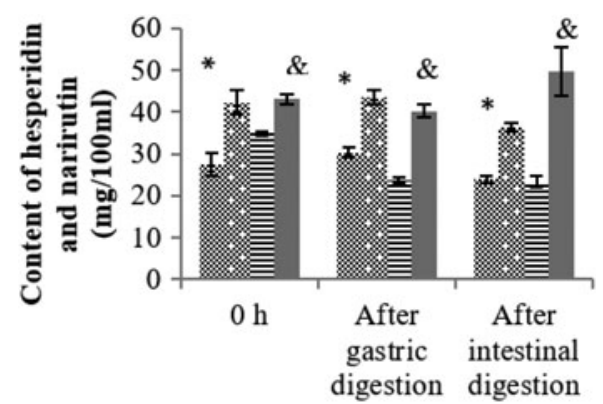

Fig. 1. Hesperidin and narirutin content of $(\mathrm{mg} / 100 \mathrm{ml})$ orange juice digestive fluids with or without $\mathrm{Y}(\mathrm{n}=3)$

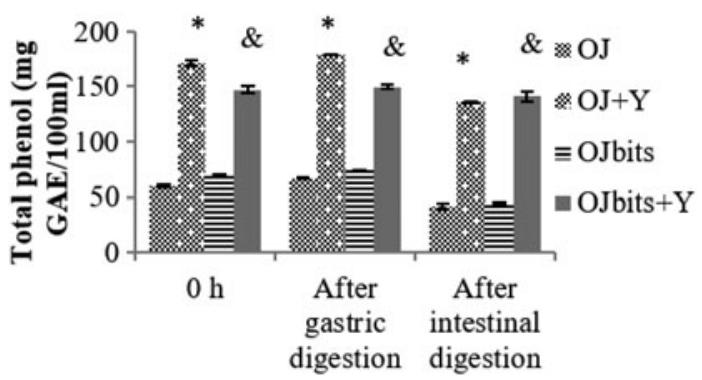

Fig. 2. Total phenols in orange juice digestive fluids $(\mathrm{mg} \mathrm{GAE} / 100 \mathrm{ml})$ with and without $\mathrm{Y}(\mathrm{n}=3)$

Yoghurt increased the amount of OJ flavanones in the digestion fluid during the upper GI digestion. This may result in more available flavanones in the colon for bacteria and would not explain the much lower urinary excretion of phenolic acids seen by Roowi et al ${ }^{(1)}$. The impact of yoghurt on bacterial metabolism in the colon therefore needs to be evaluated.

1. Roowi S, Mullen W, Edwards CA, et al. (2009). Mol Nutr Food Res. 53(S1), S68-S75. 